УДК 517.5:519.2

\title{
On some Properties of Weighted Hilbert Spaces
}

\author{
Vladislav V. Branishti* \\ Institute of Computer Science and Telecommunications \\ Reshetnev Siberian State Aerospace University \\ Krasnoyarskiy rabochiy, 31, Krasnoyarsk, 660014
}

Russia

Received 14.02.2017, received in revised form 17.05.2017, accepted 10.08.2017

We describe the weighted Hilbert spaces $L_{2, w}(\Omega)$ with positive weight functions $w(x)$ which are summable on every bounded interval. We give sufficient condition for $L_{2, w_{1}}(\Omega)$ space to be extension of $L_{2, w_{2}}(\Omega)$ space. We also describe how to use given result in statistical probability density estimation.

Keywords: integrable function spaces, Hilbert spaces, weighted function spaces, second order splines, probability density function estimating.

DOI: 10.17516/1997-1397-2017-10-4-410-421.

\section{Introduction}

Let $L_{2, w}(\Omega)$, where $w(x)$ is measurable positive function, $\Omega \subseteq \mathbb{R}$ is measurable subset, be a space of real functions $f: \Omega \rightarrow \mathbb{R}$ for which the integral

$$
\int_{\Omega} f^{2}(x) w(x) d x
$$

is finite. The measure and the integral are comprehended in Lebesgue sense. Given space is often described (e.g. [1,2]) as a case of $L_{2}(\Omega, \Sigma, \mu)$ space, where $\Sigma$ is a $\sigma$-algebra of measurable subsets of $\Omega, \mu$ is a measure on $\Sigma$, which is defined by:

$$
\mu(X)=\int_{X} w(x) d x
$$

Particularly, the space $L_{2, w}(\Omega)$ is Euclidean space with scalar product

$$
(f, g)_{w}=\int_{\Omega} f(x) g(x) w(x) d x,
$$

which induces the norm

$$
\|f\|_{2, w}=\sqrt{(f, f)_{w}} .
$$

It is also known that if the measure (1) has countable basis then the space $L_{2, w}(\Omega)$ is separable. If $w(x) \equiv 1$ then the space $L_{2, w}(\Omega)$ is denoted as $L_{2}(\Omega)$, and if $\Omega=\mathbb{R}$ then as $L_{2, w}$.

In $[3,7.1 .3]$ they consider a weighted space $L_{p}\left(R_{n}, \rho(x)\right)$, where $p \in(0 ;+\infty), R_{n}=\mathbb{R}^{n}$ is $n$-dimensional arithmetic space, and $\rho(x)>0$ is Borel measurable function on $R_{n}$. The paper [4] gives conditions on weight function $w(x)$, which makes wavelet spline system be a conditional

*branishti@sibsau.ru

(c) Siberian Federal University. All rights reserved 
or unconditional basis in $L_{p, w}(\mathbb{R})$ space, $p \in[1 ;+\infty)$. In the paper [5] they are studying similar problem for Haar wavelet system.

The $L_{2, w}(\Omega)$ space finds its application in the problem of statistical estimating of probability density function $f_{\xi}(x)$ of continuous random variate $\xi$. Indeed, in a case of completeness and separability of the space $L_{2, w}(\Omega)$ there is countable complete orthonormal system $\left\{\varphi_{j}(x)\right\}_{j=0}^{\infty}$, i.e. the system which for all function $f \in L_{2, w}(\Omega)$ satisfies the limiting relation

$$
\lim _{l \rightarrow \infty}\left\|\sum_{j=0}^{l}\left(f, \varphi_{j}\right)_{w} \varphi_{j}-f\right\|_{2, w}=0 .
$$

Thus, if $f_{\xi} \in L_{2, w}(\Omega)$ then its projective estimate $f_{l}(x)$ defined by

$$
f_{l}(x)=\sum_{j=0}^{l} \alpha_{j} \varphi_{j}(x)=\sum_{j=0}^{l}\left(f_{\xi}, \varphi_{j}\right)_{w} \varphi_{j}(x)
$$

converges to $f_{\xi}(x)$ in norm of the space $L_{2, w}(\Omega)$.

The paper [6] shows that for each continuous random variate $\xi$ and for appropriate weight function $w(x)$ there exists the space $L_{2, w}(\mathbb{R})$ including it. In so doing, the choice of the function $w(x)$ is important for convergence speed of projective estimate. In connection with it there is a necessity to investigate the properties of the $L_{2, w}(\Omega)$ spaces in the context of weight function choice.

\section{Main results}

By virtue of $\sigma$-additivity of Lebesgue integral, for each measurable positive function $w(x)$ the measure defined by (1) is also $\sigma$-additive. But if the function $w(x)$ is not summable, i.e.

$$
\int_{\Omega} w(x) d x=+\infty
$$

then it is possible the pathological behavior of the measure $\mu$ in particular cases.

For instance, let $\Omega=[0 ; 1], \Sigma$ is a $\sigma$-algebra of measurable subsets of $\Omega$ and $w(x)=\frac{1}{x}$. Then each segment from $\Sigma$ containing 0 has infinite measure:

$$
\mu[0 ; a]=\int_{0}^{a} w(t) d t=\left.\lim _{x \rightarrow+0} \ln t\right|_{x} ^{a}=+\infty, \quad a>0 .
$$

Now we take a sequence $a_{n} \downarrow 0$. Then

$$
\lim _{n \rightarrow \infty} \mu\left[0 ; a_{n}\right]=+\infty .
$$

On the other hand,

$$
\mu\left(\bigcap_{n=1}^{\infty}\left[0 ; a_{n}\right]\right)=\mu\{0\}=\int_{\{0\}} w(x) d x=0
$$

We receive that

$$
\mu\left(\bigcap_{n=1}^{\infty}\left[0 ; a_{n}\right]\right) \neq \lim _{n \rightarrow \infty} \mu\left[0 ; a_{n}\right]
$$


i.e. built measure $\mu$ is not continuous.

Further we assume that the function $w(x)$ is summable on each bounded interval $X \subset \mathbb{R}$ :

$$
\int_{X} w(x) d x<+\infty .
$$

Now, it is obvious that the measure $\mu$ induced by the function $w(x)$ is $\sigma$-finite. Then the theorem about completeness of $L_{p}(\Omega, \Sigma, \mu)$ spaces with $p \in[1 ;+\infty)$ and $\sigma$-finite measure $\mu[2, I V, 3.3]$ leads to completeness of the space $L_{2, w}(\Omega)$.

Besides the measure $\mu$ has a countable basis consisted of, for example, elements from $\sigma$ ring generated by semiring of half-intervals on real axis with rational endpoints. This leads to separability of the space $L_{2, w}(\Omega)$.

Thus, for each positive function $w(x)$ which is summable on every bounded interval the $L_{2, w}(\Omega)$ space is separable Hilbert space.

Present paper considers relationship between $L_{2, w}(\Omega)$ spaces with common set $\Omega$ and different weight functions $w(x)$.

Definition. The space $L_{2, w_{1}}(\Omega)$ is called an extension of the space $L_{2, w_{2}}(\Omega)$ if the strict inclusion

$$
L_{2, w_{2}}(\Omega) \subset L_{2, w_{1}}(\Omega)
$$

holds.

Let us denote obvious proposition.

Proposition 1. If the inequality $w_{1}(x) \leqslant w_{2}(x)$ holds for all $x$ from $\Omega$, then

$$
L_{2, w_{2}}(\Omega) \subseteq L_{2, w_{1}}(\Omega)
$$

Particularly, if $w(x) \leqslant 1$ then the space $L_{2, w}(\Omega)$ includes the space $L_{2}(\Omega)$.

Proof. It follows from $w_{1}(x) \leqslant w_{2}(x)$ that for all functions $f: \Omega \rightarrow \mathbb{R}$ we have

$$
f^{2}(x) w_{1}(x) \leqslant f^{2}(x) w_{2}(x),
$$

and

$$
\int_{\Omega} f^{2}(x) w_{1}(x) d x \leqslant \int_{\Omega} f^{2}(x) w_{2}(x) d x .
$$

Now, convergence of the integral at the left side follows from convergence of the integral at the right one. Thus, for all function $f: \Omega \rightarrow \mathbb{R}$ we have that $f \in L_{2, w_{2}}(\Omega)$ involves $f \in L_{2, w_{1}}(\Omega)$, i.e.

$$
L_{2, w_{2}}(\Omega) \subseteq L_{2, w_{1}}(\Omega)
$$

Remark 1. The conclusion of the Proposition 1 remains true even when the inequality $w_{1}(x) \leqslant$ $w_{2}(x)$ holds almost everywhere on $\Omega$.

Proposition 2. With introduced assumptions on weighted functions $w_{1}(x)$ and $w_{2}(x)$ it is true that

$$
L_{2, w_{1}}(\Omega) \cap L_{2, w_{2}}(\Omega)=L_{2, w_{\max }}(\Omega),
$$

where $w_{\max }(x)=\max \left\{w_{1}(x), w_{2}(x)\right\}$. 
Proof. It is obvious that the function $w_{\max }(x)$ is also summable on each bounded interval. Then the space $L_{2, w_{\max }}(\Omega)$ is defined and separable Hilbert. At the same time $w_{\max }(x) \geqslant w_{1}(x)$ and $w_{\max }(x) \geqslant w_{2}(x)$. Then from Proposition 1 we have that

$$
L_{2, w_{\max }}(\Omega) \subseteq L_{2, w_{1}}(\Omega) \quad \text { and } \quad L_{2, w_{\max }}(\Omega) \subseteq L_{2, w_{2}}(\Omega)
$$

i.e.

$$
L_{2, w_{\max }}(\Omega) \subseteq L_{2, w_{1}}(\Omega) \cap L_{2, w_{2}}(\Omega) .
$$

To prove inverse inclusion we can take arbitrary function $f(x)$ from the set $L_{2, w_{1}}(\Omega) \cap$ $L_{2, w_{2}}(\Omega)$. From definition of the space $L_{2, w}(\Omega)$ we will have:

$$
\int_{\Omega} f^{2}(x) w_{1}(x) d x<+\infty \text { and } \int_{\Omega} f^{2}(x) w_{2}(x) d x<+\infty .
$$

Let us split the space $\Omega$ by two subsets $\Omega_{1}$ and $\Omega_{2}$, where

$$
\begin{gathered}
\Omega_{1}=\left\{x \in \Omega \mid w_{1}(x) \geqslant w_{2}(x)\right\}, \\
\Omega_{2}=\Omega \backslash \Omega_{1}=\left\{x \in \Omega \mid w_{1}(x)<w_{2}(x)\right\} .
\end{gathered}
$$

Then

$$
\int_{\Omega} f^{2}(x) w_{1}(x) d x=\int_{\Omega_{1}} f^{2}(x) w_{1}(x) d x+\int_{\Omega_{2}} f^{2}(x) w_{1}(x) d x .
$$

We have got that both of the integrals

$$
\int_{\Omega_{1}} f^{2}(x) w_{1}(x) d x \text { and } \int_{\Omega_{2}} f^{2}(x) w_{1}(x) d x
$$

exist and are finite.

Similarly, folowing integrals exist and are finite:

$$
\int_{\Omega_{1}} f^{2}(x) w_{2}(x) d x \text { and } \int_{\Omega_{2}} f^{2}(x) w_{2}(x) d x .
$$

Now we will consider the sum of the integrals $\int_{\Omega_{1}} f^{2}(x) w_{1}(x) d x$ and $\int_{\Omega_{2}} f^{2}(x) w_{2}(x) d x$ :

$$
\begin{gathered}
\int_{\Omega_{1}} f^{2}(x) w_{1}(x) d x+\int_{\Omega_{2}} f^{2}(x) w_{2}(x) d x= \\
=\int_{\Omega_{1}} f^{2}(x) w_{\max }(x) d x+\int_{\Omega_{2}} f^{2}(x) w_{\max }(x) d x=\int_{\Omega} f^{2}(x) w_{\max }(x) d x<+\infty .
\end{gathered}
$$

We have from this that $f \in L_{2, w_{\max }}(\Omega)$. So the inclusion

$$
L_{2, w_{1}}(\Omega) \cap L_{2, w_{2}}(\Omega) \subseteq L_{2, w_{\max }}(\Omega),
$$

is proved and the conclusion of the proposition as well.

The paper [6] gives necessary condition on weight functions $w_{1}(x)$ and $w_{2}(x)$ to spaces $L_{2, w_{1}}(\Omega)$ and $L_{2, w_{2}}(\Omega)$ not be equal. We express here a stronger proposition.

Proposition 3. If $L_{2, w_{1}}(\Omega) \neq L_{2, w_{2}}(\Omega)$, then at least one of the inequalities holds:

$$
\operatorname{ess} \inf _{x \in \Omega} \frac{w_{1}(x)}{w_{2}(x)}=0 \quad \text { or } \quad \operatorname{ess} \sup _{x \in \Omega} \frac{w_{1}(x)}{w_{2}(x)}=+\infty \text {. }
$$


Proof. On the contrary we assume that all inequalities (2) do not hold. Then

$$
\operatorname{ess} \inf _{x \in \Omega} \frac{w_{1}(x)}{w_{2}(x)}=m>0, \quad \operatorname{ess} \sup _{x \in \Omega} \frac{w_{1}(x)}{w_{2}(x)}=M<\infty ;
$$

i.e. almost everywhere on $\Omega$

$$
0<m \leqslant \frac{w_{1}(x)}{w_{2}(x)} \leqslant M<+\infty
$$

It follows from the given inequalities that almost everywhere on $\Omega$

$$
w_{1}(x) \leqslant M w_{2}(x), \quad w_{2}(x) \leqslant \frac{1}{m} w_{1}(x) .
$$

Then

$$
\begin{aligned}
& \int_{\Omega} f^{2}(x) w_{1}(x) d x \leqslant \int_{\Omega} f^{2}(x) M w_{2}(x) d x=M \int_{\Omega} f^{2}(x) w_{2}(x) d x \\
& \int_{\Omega} f^{2}(x) w_{2}(x) d x \leqslant \int_{\Omega} f^{2}(x) \frac{1}{m} w_{1}(x) d x=\frac{1}{m} \int_{\Omega} f^{2}(x) w_{1}(x) d x
\end{aligned}
$$

We have now that (3) leads to inclusion $L_{2, w_{1}}(\Omega) \subseteq L_{2, w_{2}}(\Omega)$, and (4) leads to $L_{2, w_{2}}(\Omega) \subseteq$ $L_{2, w_{1}}(\Omega)$.

It follows from the Proposition 3 that if $L_{2, w_{1}}(\Omega)$ is an extension for $L_{2, w_{2}}(\Omega)$, then

$$
\text { ess } \inf _{x \in \Omega} \frac{w_{1}(x)}{w_{2}(x)}=0 .
$$

Let we give sufficient condition for $L_{2, w_{1}}(\Omega)$ to contain elements which are outside of $L_{2, w_{2}}(\Omega)$.

Theorem 1. Let $\Omega \subseteq \mathbb{R}$ contains right-side or left-side neighborhood of some point $a \in \mathbb{R}, w_{1}(x)$ and $w_{2}(x)$ are positive on $\Omega$ functions which are summable on every bounded interval and for which at least one of one-sided limits

$$
\lim _{x \rightarrow a+0} \frac{w_{1}(x)}{w_{2}(x)} \quad \text { or } \quad \lim _{x \rightarrow a-0} \frac{w_{1}(x)}{w_{2}(x)}
$$

is equal 0 . Then

$$
L_{2, w_{1}}(\Omega) \backslash L_{2, w_{2}}(\Omega) \neq \varnothing .
$$

Proposition 1 and Theorem 1 lead to convenient sufficient condition for extension of the space $L_{2, w}(\Omega)$. Let

1) $\Omega$ contains right-side or left-side neighborhood of some point $a \in \mathbb{R}$;

2) $w_{1}(x) \leqslant w_{2}(x)$ holds almost everywhere on $\Omega$;

3) $\lim _{x \rightarrow a+0} \frac{w_{1}(x)}{w_{2}(x)}=0$ or $\lim _{x \rightarrow a-0} \frac{w_{1}(x)}{w_{2}(x)}=0$.

Then

$$
L_{2, w_{2}}(\Omega) \subset L_{2, w_{1}}(\Omega)
$$




\section{Proof of the Theorem 1}

We have to prove some intermediate propositions before we prove the Theorem 1 .

Lemma 1. Let $\Omega=(A ;+\infty)$, where $A \in[-\infty ;+\infty)$ and $f(x)$ is differentiable positive nonincreasing on $\Omega$ function which satisfies

$$
\lim _{x \rightarrow+\infty} f(x)=0 .
$$

Then there exists non-negative on $\Omega$ function $g(x)$, for which

$$
\int_{\Omega} g(x) d x=+\infty \quad \text { and } \quad \int_{\Omega} f(x) g(x) d x<+\infty .
$$

Proof. We define the function $g(x)$ on $\Omega$ in this way:

$$
g(x)=-\frac{f^{\prime}(x)}{f(x)} .
$$

Because of $f(x)>0$ and $f^{\prime}(x) \leqslant 0$ then $g(x) \geqslant 0$. Further,

$$
\begin{gathered}
\int_{\Omega} g(x) d x=-\int_{A}^{+\infty} \frac{f^{\prime}(x)}{f(x)} d x=\ln f(A)-\lim _{x \rightarrow+\infty} \ln f(x)=+\infty \\
\int_{\Omega} f(x) g(x) d x=-\int_{A}^{+\infty} f^{\prime}(x) d x=f(A)-\lim _{x \rightarrow+\infty} f(x)=f(A)<+\infty .
\end{gathered}
$$

Thus, function $g(x)$ satisfies the conclusion of the lemma.

Lemma 2. The conclusion of the lemma 1 remains true if in the condition we change differentiability of the function $f(x)$ by its piecewise constancy on $\Omega$.

Proof. Let the function $f(x)$ is piecewise constant, positive and does not increase on $\Omega$. Then $\Omega$ can be split by points

$$
A=x_{0}<x_{1}<\cdots<x_{n}<\cdots
$$

to intervals

$$
\left(x_{0} ; x_{1}\right),\left(x_{1} ; x_{2}\right), \ldots,\left(x_{n-1} ; x_{n}\right), \ldots
$$

in which the function $f(x)$ is constant:

$$
f(x)=y_{n}, \quad x \in\left(x_{n-1} ; x_{n}\right), \quad n=1,2, \ldots
$$

In this case

$$
y_{1}>y_{2}>\cdots>y_{n}>\cdots
$$

and

$$
\lim _{n \rightarrow \infty} y_{n}=0 .
$$

We are going to prove that for the function $f(x)$ there exists a majorizing function $f_{0}(x)$, i.e.

$$
f(x) \leqslant f_{0}(x), \quad x \in \Omega,
$$

which satisfies the condition of the Lemma 1. 
We can build the function $f_{0}(x)$ in the form of 2 nd order infinity spline passing through the points $\left(x_{1}, y_{1}\right),\left(x_{2}, y_{2}\right), \ldots$ :

$$
f_{0}(x)= \begin{cases}s_{0}(x), & x \in\left(x_{0} ; x_{1}\right] \\ s_{1}(x), & x \in\left(x_{1} ; x_{2}\right] \\ \vdots & \\ s_{n}(x), & x \in\left(x_{n} ; x_{n+1}\right] \\ \vdots & \end{cases}
$$

Each of the functions $s_{n}(x)$ is a 2 nd order polynomial:

$$
s_{n}(x)=a_{n} x^{2}+b_{n} x+c_{n}, \quad x \in\left(x_{n} ; x_{n+1}\right]
$$

To reach a continuity and smoothness of the function $f_{0}(x)$ over all set $\Omega$ we submit the functions $s_{n}(x)$ to next conditions:

$$
\left\{\begin{array}{l}
s_{n}\left(x_{n}\right)=y_{n} \\
s_{n}\left(x_{n+1}\right)=y_{n+1} \\
s_{n}^{\prime}\left(x_{n}\right)=s_{n-1}^{\prime}\left(x_{n}\right)
\end{array}, \quad n=1,2, \ldots\right.
$$

At that for $s_{0}(x)$ we can take

$$
s_{0}(x) \equiv y_{1} .
$$

We are going to show that the system (7) defines unique 2 nd order polynomial $s_{n}(x)$ for all $x_{n}, x_{n+1}, y_{n}, y_{n+1}$ and $s_{n-1}^{\prime}\left(x_{n}\right)=y_{n}^{\prime}$ satisfying the conditions:

$$
x_{n}<x_{n+1}, \quad y_{n}>y_{n+1} .
$$

Indeed, the system (7) leads to system of linear equations with variable coefficients $a_{n}, b_{n}$ and $c_{n}$ :

$$
\left\{\begin{array}{l}
a_{n} x_{n}^{2}+b_{n} x_{n}+c_{n}=y_{n} \\
a_{n} x_{n+1}^{2}+b_{n} x_{n+1}+c_{n}=y_{n+1} \\
2 a_{n} x_{n}+b_{n}=y_{n}^{\prime}
\end{array}\right.
$$

The determinant of basic matrix of this system is

$$
\left|\begin{array}{ccc}
x_{n}^{2} & x_{n} & 1 \\
x_{n+1}^{2} & x_{n+1} & 1 \\
2 x_{n} & 1 & 0
\end{array}\right|=\left(x_{2}-x_{1}\right)^{2}>0
$$

so the system has a unique solution:

$$
a_{n}=\frac{\Delta_{1}}{\left(x_{2}-x_{1}\right)^{2}}, \quad b_{n}=\frac{\Delta_{2}}{\left(x_{2}-x_{1}\right)^{2}}, \quad c_{n}=\frac{\Delta_{3}}{\left(x_{2}-x_{1}\right)^{2}},
$$

where

$$
\begin{gathered}
\Delta_{1}=\left|\begin{array}{ccc}
y_{n} & x_{n} & 1 \\
y_{n+1} & x_{n+1} & 1 \\
y_{n}^{\prime} & 1 & 0
\end{array}\right|, \quad \Delta_{2}=\left|\begin{array}{ccc}
x_{n}^{2} & y_{n} & 1 \\
x_{n+1}^{2} & y_{n+1} & 1 \\
2 x_{n} & y_{n}^{\prime} & 0
\end{array}\right|, \quad \Delta_{3}=\left|\begin{array}{ccc}
x_{n}^{2} & x_{n} & y_{n} \\
x_{n+1}^{2} & x_{n+1} & y_{n+1} \\
2 x_{n} & 1 & y_{n}^{\prime}
\end{array}\right| \\
-416-
\end{gathered}
$$


Further, in order to make the spline $f_{0}(x)$ satisfy the condition of majority $(6)$ it is necessary and sufficient to satisfy

$$
s_{n}(x) \geqslant y_{n+1}, \quad x \in\left(x_{n} ; x_{n+1}\right), \quad n=0,1, \ldots
$$

Last condition will hold if $s_{n}^{\prime}\left(x_{n+1}\right) \leqslant 0$, i.e. $2 a_{n} x_{n+1}+b_{n} \leqslant 0$.

When we substitute in this inequality the solution (8), we will have

$$
y_{n}^{\prime} \geqslant \frac{2\left(y_{n+1}-y_{n}\right)}{x_{n+1}-x_{n}} .
$$

In the case

$$
y_{n}^{\prime}<\frac{2\left(y_{n+1}-y_{n}\right)}{x_{n+1}-x_{n}},
$$

we will build the function $s_{n}(x)$ by this way:

$$
s_{n}(x)=\left\{\begin{array}{ll}
s_{n}^{(1)}(x) & x \in\left(x_{n} ; t\right] \\
s_{n}^{(2)}(x) & x \in\left(t ; x_{n+1}\right]
\end{array},\right.
$$

where $s_{n}^{(1)}(x)$ and $s_{n}^{(2)}(x)$ are 2nd order polynomials

$$
\begin{aligned}
& s_{n}^{(1)}(x)=a_{n}^{(1)} x^{2}+b_{n}^{(1)} x+c_{n}^{(1)}, \\
& s_{n}^{(2)}(x)=a_{n}^{(2)} x^{2}+b_{n}^{(2)} x+c_{n}^{(2)},
\end{aligned}
$$

which are defined by this conditions:

$$
\left\{\begin{array}{l}
s_{n}^{(1)}\left(x_{n}\right)=y_{n} \\
\left.\frac{d}{d x} s_{n}^{(1)}(x)\right|_{x=x_{n}}=y_{n}^{\prime} \\
s_{n}^{(1)}(t)=\frac{1}{2}\left(y_{n}+y_{n+1}\right) \\
\left.\frac{d}{d x} s_{n}^{(1)}(x)\right|_{x=t}=0
\end{array},\left\{\begin{array}{l}
s_{n}^{(2)}(t)=\frac{1}{2}\left(y_{n}+y_{n+1}\right) \\
s_{n}^{(2)}\left(x_{n+1}\right)=y_{n+1} \\
\left.\frac{d}{d x} s_{n}^{(2)}(x)\right|_{x=t}=0
\end{array}, \quad x_{n}<t<x_{n+1}\right.\right.
$$

(see Fig. 1).

The second system in (10) is similar to the system (7), therefore it defines unique function $s_{n}^{(2)}(x)$.

If we substitute the expression for $s_{n}^{(1)}(x)$ in $(10)$, we will get (after exclusion $t$ ):

$$
\left\{\begin{array}{l}
a_{n}^{(1)} x_{n}^{2}+b_{n}^{(1)}+c_{n}^{(1)}=y_{n} \\
2 a_{n}^{(1)} x_{n}+b_{n}^{(1)}=y_{n}^{\prime} \\
4 a_{n}^{(1)} c_{n}^{(1)}-\left(b_{n}^{(1)}\right)^{2}=\frac{1}{2} a_{n}^{(1)}\left(y_{n}+y_{n+1}\right)
\end{array} .\right.
$$

The last system is not linear but we can get unique solution by elementary simplifying:

$$
\left\{\begin{array}{l}
a_{n}^{(1)}=\frac{\left(y_{n}^{\prime}\right)^{2}}{2\left(y_{n}-y_{n+1}\right)} \\
b_{n}^{(1)}=y_{n}^{\prime}-2 a_{n}^{(1)} x_{n} \\
c_{n}^{(1)}=y_{n}+a_{n}^{(1)} x_{n}^{2}-x_{n} y_{n}^{\prime}
\end{array} .\right.
$$




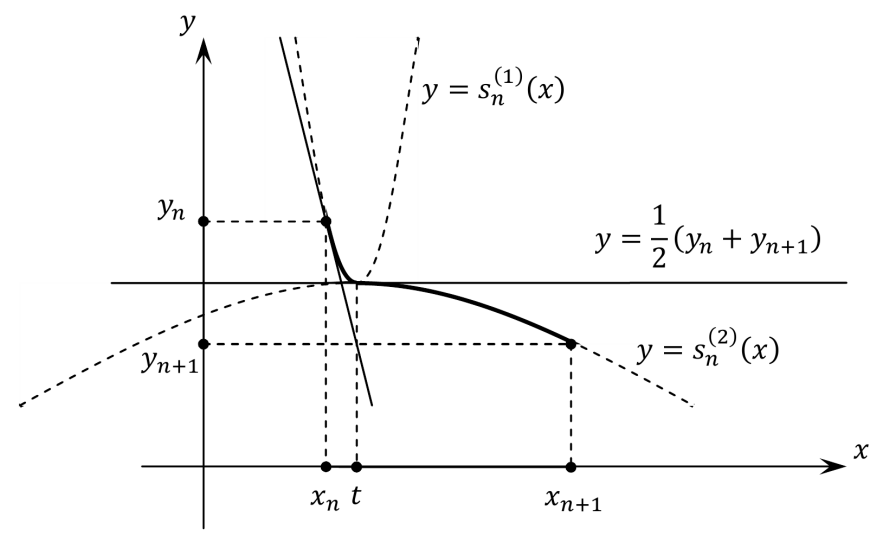

Fig. 1. Building of the function $s_{n}(x)$ in the case (9)

Now we check whether found solution satisfies to inequality in (10). From the first system we find $t$ :

$$
t=-\frac{b_{n}^{(1)}}{2 a_{n}^{(1)}}=x_{n}-\frac{y_{n}-y_{n+1}}{y_{n}^{\prime}} .
$$

At the same time because of $y_{n}-y_{n+1}>0$ and $y_{n}^{\prime}<\frac{2\left(y_{n+1}-y_{n}\right)}{x_{n+1}-x_{n}}<0$, then $t>x_{n}$. Further,

$$
t=x_{n}-\frac{y_{n}-y_{n+1}}{y_{n}^{\prime}}<x_{n}-\frac{y_{n}-y_{n+1}}{\frac{2\left(y_{n+1}-y_{n}\right)}{x_{n+1}-x_{n}}}=\frac{x_{n}+x_{n+1}}{2}<x_{n+1} \text {. }
$$

Thus, part $s_{n}(x)$ of the spline $f_{0}(x)$ in the case of $(9)$ is also built. We have that whole spline $f_{0}(x)$ is smooth on $\Omega$, passes through the points $\left(x_{1}, y_{1}\right),\left(x_{2}, y_{2}\right), \ldots$ and satisfies $(6)$.

We will show that the function $f_{0}(x)$ satisfies the condition of the Lemma 1. First, $f_{0}(x)$ is differentiable on $\Omega$. Second, $f_{0}(x)$ is positive because of $f_{0}(x) \geqslant f(x)>0$. Third, according to building we have $f_{0}^{\prime}(x) \leqslant 0$, therefore the function $f_{0}(x)$ does not increase.

Last, for all $x \in\left(x_{n} ; x_{n+1}\right)$ the following holds: $f_{0}(x) \leqslant y_{n}$, and so

$$
\begin{gathered}
0 \leqslant \lim _{x \rightarrow+\infty} f_{0}(x) \leqslant \lim _{n \rightarrow \infty} y_{n}=0 ; \\
\lim _{x \rightarrow+\infty} f_{0}(x)=0 .
\end{gathered}
$$

Then it follows from Lemma 1 that there exists non-negative function $g(x)$, for which

$$
\int_{\Omega} g(x) d x=+\infty \quad \text { and } \quad \int_{\Omega} f_{0}(x) g(x) d x<+\infty .
$$

Finiteness of the first integral and the inequality (6) lead to that the integral

$$
\int_{\Omega} f(x) g(x) d x
$$

is finite.

Thus, the function $g(x)$ satisfies the conclusion of the Lemma 2. 
Lemma 3. The conclusion of the Lemma 1 is true for all positive function $f(x)$, for which

$$
\lim _{x \rightarrow+\infty} f(x)=0 .
$$

Proof. Let function $f(x)$ satisfies to the condition of the Lemma 3. According to definition of limit of function, for all $\varepsilon>0$ there exists $M \in \Omega$ for which for all $x>M$ following holds:

$$
f(x)<\varepsilon .
$$

Now we take a sequence $\varepsilon_{n}=\frac{1}{n}$. Some sequence $M_{n}$ corresponds to it. Let us to consider a function

$$
f_{0}(x)= \begin{cases}1, & x \in\left[M_{1} ; M_{2}\right) \\ \frac{1}{2}, & x \in\left[M_{2} ; M_{3}\right) \\ \vdots & \\ \frac{1}{n}, & x \in\left[M_{n} ; M_{n+1}\right) \\ \vdots & \end{cases}
$$

This function satisfies the condition of the Lemma 2. Therefore, there exists non-negative function $g(x)$, for which

$$
\int_{\Omega} g(x) d x=+\infty \quad \text { and } \quad \int_{\Omega} f_{0}(x) g(x) d x<+\infty .
$$

It is obvious that on $\Omega$ the inequality $f(x) \leqslant f_{0}(x)$ holds. Then the integral

$$
\int_{\Omega} f(x) g(x) d x
$$

is finite.

Lemma 4. Let $\Omega=(a ; b)$ and $f(x)$ is positive on $\Omega$ function for which at least one of single-sided limit

$$
\lim _{x \rightarrow a+0} f(x) \quad \text { or } \quad \lim _{x \rightarrow b-0} f(x)
$$

is equal 0. Then there exists non-negative on $\Omega$ function $h(x)$, for which

$$
\int_{\Omega} h(x) d x=+\infty, \quad \int_{\Omega} f(x) h(x) d x<+\infty .
$$

Proof. Let us to consider the case of right-sided limit. We define a variable $y=\frac{1}{x-a}$.

Then

$$
\begin{gathered}
x \rightarrow a+0 \quad \text { is equivalent to } \quad y \rightarrow+\infty \\
x=b \quad \text { is equivalent to } \quad y=\frac{1}{b-a} \\
f(x)=f\left(a+\frac{1}{y}\right)
\end{gathered}
$$

and the function $f\left(a+\frac{1}{y}\right)$ (from variable $y$ ) defined on $\Omega^{\prime}=\left(\frac{1}{b-a} ;+\infty\right)$ satisfies the condition of the Lemma 3. Then there exists the function $g(x)$, for which

$$
\int_{\Omega^{\prime}} g(y) d y=+\infty \quad \text { and } \quad \int_{\Omega^{\prime}} f\left(a+\frac{1}{y}\right) g(y) d y<+\infty .
$$


Because of

$$
\begin{gathered}
\int_{\Omega^{\prime}} g(y) d y=\int_{\Omega^{\prime}} g\left(\frac{1}{x-a}\right) \frac{1}{(x-a)^{2}} d x=+\infty \\
\int_{\Omega^{\prime}} f\left(a+\frac{1}{y}\right) g(y) d y=\int_{\Omega^{\prime}} f(x) g\left(\frac{1}{x-a}\right) \frac{1}{(x-a)^{2}} d x<+\infty
\end{gathered}
$$

we can take for function $h(x)$

$$
h(x)=g\left(\frac{1}{x-a}\right) \frac{1}{(x-a)^{2}} .
$$

The case of left-sided limit is considered similar: $y=\frac{1}{b-x}$, and

$$
h(x)=-g\left(\frac{1}{b-x}\right) \frac{1}{(b-x)^{2}}
$$

Proof of Theorem 1. We are going to prove that in the $L_{2, w_{1}}(\Omega)$ space there is a function $f$ which does not belong the $L_{2, w_{2}}(\Omega)$ space, i.e.

$$
\int_{\Omega} f^{2}(x) w_{1}(x) d x<+\infty \quad \text { and } \quad \int_{\Omega} f^{2}(x) w_{2}(x) d x=+\infty .
$$

The function $\frac{w_{1}(x)}{w_{2}(x)}$ satisfies the condition of the Lemma 4. Then there exists non-negative on $\Omega$ function $h(x)$, for which

$$
\int_{\Omega} h(x) d x=+\infty, \quad \int_{\Omega} h(x) \frac{w_{1}(x)}{w_{2}(x)} d x<+\infty .
$$

We define the required function $f(x)$ by this way: $f(x)=\sqrt{\frac{h(x)}{w_{2}(x)}}$.

We get:

$$
\begin{gathered}
\int_{\Omega} f^{2}(x) w_{2}(x) d x=\int_{\Omega} h(x) d x=+\infty \\
\int_{\Omega} f^{2}(x) w_{1}(x) d x=\int_{\Omega} h(x) \frac{w_{1}(x)}{w_{2}(x)} d x<+\infty .
\end{gathered}
$$

\section{Conclusion}

Present paper describes the properties of weighted functional Hilbert spaces of $L_{2, w}(\Omega)$ kind in the context of building probability density function estimate for continuous random variable $\xi$. Proposition about convergence of probability density function projective estimate is true in assumption that the probability density belongs to the space $L_{2, w}(\Omega)$ with appropriate weight function $w(x)$. However, the situations when that information is absent can appear in applications. The Theorem 1 of present paper suggests particularly the method of choice required function $w(x)$. For instance, if according to the received values of random variate $\xi$ being investigated we have reasons to assume that for the chosen weight function $w_{2}(x)$ the equality

$$
\|f\|_{w_{2}}^{2}=\int_{\Omega} f^{2}(x) w_{2}(x) d x=+\infty
$$


holds, i.e. $f \notin L_{2, w_{2}}(\Omega)$, then we can try to extend the space $\int_{\Omega} f^{2}(x) w_{2}(x) d x$ to space $\int_{\Omega} f^{2}(x) w_{1}(x) d x$ by taking the function $w_{1}(x)$ satisfied condition:

$$
\lim _{x \rightarrow a} \frac{w_{1}(x)}{w_{2}(x)}=0
$$

e.g.

$$
w_{1}(x)=\left\{\begin{array}{ll}
|x-a|^{\alpha} w_{2}(x), & x \in(a-\varepsilon ; a+\varepsilon) \\
w_{2}(x), & \text { else }
\end{array}, \alpha>0, \varepsilon \in(0 ; 1],\right.
$$

and the point $a$ is chosen from the condition

$$
\int_{a-\delta}^{a+\delta} f^{2}(x) w_{2}(x) d x=+\infty \quad \text { for all } \delta>0
$$

\section{References}

[1] A.N.Kolmogorov, S.V.Fomin, Elements of the Theory of Functions and Functional Analysis, Dover Publications, Inc., 1999.

[2] L.V.Kantorovich, G.P.Akilov, Functional Analysis, Pergamon Press, 1982.

[3] H.Triebel, Theory of function spaces, Akademische Verlagsgesellschaft Geest \& Portig K.-G., Leipzig, 1983.

[4] J.Garcia-Cuerva, K.S.Kazarian, Spline wavelet bases of weighted $L^{p}$ spaces, $1 \leqslant p<\infty$, Proceedings of the American Mathematical Society, 123(1995), no. 2, 433-439.

[5] K.S.Kazarian, S.S.Kazaryan, A.San Antolín, Wavelets in weighted norm spaces, arXiv: 1410.4888, 2014.

[6] V.V.Branishti, Introducing the $L_{2, w}$ space for building the projective estimation of probability density function, Vestnik SibGAU., 17, 1(2016), 19-26 (in Russian).

\section{О некоторых свойствах весовых гильбертовых пространств}

Владислав В.Браништи

Институт информатики и телекоммуникаций Сибирский государственный аэрокосмический университет Красноярский рабочий, 31, Красноярск, 660014

Россия

В работе рассматриваются весовые гилъбертовы пространства $L_{2, w}(\Omega)$ при положительных $u$ суммируемых на любом ограниченном интервале весовых функииях w(x). Приводится достаточное условие, при котором пространство $L_{2, w_{1}}(\Omega)$ является расширением пространства $L_{2, w_{2}}(\Omega)$. Описъвается применение полученного результата при статистическом оценивании функиии плотности вероятности случайной величины.

Ключевые слова: пространства интегрируемых бункций, гилъбертовъ пространства, весовъе функционалъные пространства, сплайны второго порядка, оценивание функции плотности вероятности. 